\title{
openheart Clinical governance programme in patients with acute coronary syndrome: design and methodology of a quality improvement initiative
}

\author{
Sergio Leonardi (D) , ${ }^{1,2}$ Claudio Montalto, ${ }^{1}$ Gianni Casella, ${ }^{3}$ Daniele Grosseto, ${ }^{4}$ \\ Alessandra Repetto, ${ }^{2}$ Monica Portolan, ${ }^{2}$ Federico Fortuni, ${ }^{1}$ Filippo Ottani, ${ }^{5}$ \\ Marcello Galvani, ${ }^{5}$ Laura Sofia Cardelli, ${ }^{6,7}$ Stefano De Servi, ${ }^{1,8}$ Andrea Rubboli, ${ }^{9}$ \\ Gaetano Maria De Ferrari, ${ }^{10}$ Luigi Oltrona Visconti, ${ }^{2}$ Gianluca Campo (1) ${ }^{7}$
}

\begin{abstract}
- Additional material is published online only. To view, please visit the journal online (http://dx.doi.org/10.1136/ openhrt-2020-001415)
\end{abstract}

To cite: Leonardi S, Montalto C Casella G, et al. Clinical governance programme in patients with acute coronary syndrome: design and methodology of a quality improvement initiative. Open Heart 2020;7:e001415.

doi:10.1136/

openhrt-2020-001415

Received 11 August 2020 Revised 28 September 2020 Accepted 2 October 2020
Check for updates

(C) Author(s) (or their employer(s)) 2020. Re-use permitted under CC BY-NC. No commercial re-use. See rights and permissions. Published by BMJ.

For numbered affiliations see end of article.

Correspondence to

Dr Sergio Leonardi; s.leonardi@ smatteo.pv.it

\section{ABSTRACT}

Introduction Despite the availability of diverse evidencebased diagnostic and treatment options, many patients with acute coronary syndrome (ACS) still fail to receive effective, safe and timely diagnoses and therapies. The Association of Acute CardioVascular Care of the European Society of Cardiology has proposed and retrospectively validated a set of ACS-specific quality indicators. Combining these indicators with the principles of clinical governance-a holistic, patient-centred approach intended to promote continuous quality improvement-we designed the clinical governance programme in patients with ACS.

Methods and analysis This is a multicentre quality improvement initiative exploring multiple dimensions of care, including diagnosis, therapy, patient satisfaction, centre organisation and efficiency in all comers patients with ACS.

The study will enrol $\approx 5000$ patients prospectively (ie, at the time of the first objective qualifying ACS criterion) with a 1-year follow-up. Consecutive inclusion will be promoted by a simplified informed consent process and quantified by the concordance with corresponding hospital administrative records using diagnosis-related group codes of ACS.

Coprimary outcome measures are (1) timely reperfusion in patients with ST-elevation ACS and (2) optimal medical therapy at discharge in patients with confirmed acute myocardial infarction. Secondary outcomes broadly include multiple indicators of the process of care. Clinical endpoints (ie, death, myocardial infarction, stroke and bleeding) will be adjudicated by a clinical event committee according to predefined criteria.

Ethics and dissemination The study has been approved by local ethics committee of all study sites. As a quality improvement initiative and to promote consecutive inclusion of the population of interest, a written informed consent will be requested only to patients who are discharged alive. Dissemination will be actively promoted by (1) the registration site (ClinicalTrials.Gov ID NCT04255537), (2) collaborations with investigators through open data access and sharing.

\section{Key questions}

What is already known about this subject?

- Acute coronary syndrome remains a leading cause of mortality and morbidity worldwide

What does this study add?

- A study strength is a design that promote integration with clinical care, resulting in the enrolment of consecutive patients that may closely reflect the population of interest with acute coronary syndrome, including very high-risk patients who die early after presentation and those with a final diagnosis alternative to myocardial infarction or unstable angina.

How might this impact on clinical practice?

- The assessment of multiple domains of care may allow to comprehensively and accurately explore the health status and process of care of a complex clinical syndrome such as acute coronary syndrome with potential profound implications on clinical practice.

\section{RATIONALE}

To improve cardiovascular health, it is essential to translate scientific evidence into clinical practice. Therefore, patient care needs to be accountable, measured and shared. The care of patients with acute coronary syndrome (ACS), a leading cause of death and disability worldwide, has evolved and advanced dramatically in the past decades. This improvement is due to several factors, including the availability of innovative diagnostic and treatments options and more consistent use of evidenced-based therapies. However, many patients with ACS still fail to receive effective, safe and timely diagnoses and therapies. Thus, the extent to which medical advances impact patients care in modern medicine. as well as the gaps in optimal care delivery, remains only partially explored. 
Permanent clinical quality improvements programmes have been successfully developed in several countries ${ }^{1-3}$ and offer a unique opportunity both to understand how care is being delivered in practice and to promote improved quality of care (QoC). However, measuring QoC is difficult and it cannot be estimated solely on the basis of patients' clinical outcomes. ${ }^{4}$ Thus, measuring the process of care through quality indicators (QIs) has become increasingly important. The Association of Acute CardioVascular Care of the European Society of Cardiology has proposed and retrospectively validated a set of ACS-specific QIs with the intention of facilitating programme to improve QoC for patients with ACS across Europe. ${ }^{5-7}$

In Italy, some studies with this goal have been conducted by national cardiovascular societies. ${ }^{8-10}$ However, their interpretation has been challenged, to various extent by: (1) the enrolment of time-limited cohorts (mostly with cross-sectional design), (2) the uncertain consecutiveness of included patients, (3) the lack of systematic adjudication of study outcome, (4) a limited assessment of the numerous dimensions of care integrating diagnostic, therapeutic and systemlevel domains and finally (5) the exclusion of patients with ACS at admission but without myocardial infarction (MI) or unstable angina (UA) at discharge. Also data sharing, an essential component of initiatives primarily intended to serve patients, has been very limited.

Herein, we present the design of the Clinical Governance Programme in patients with ACS, a quality improvement initiative designed to fill these gaps with the ultimate goal to inform a permanent national surveillance initiative aimed to improve the QoC of patients with ACS.

\section{DESIGN}

This programme is a prospective, observational study designed to include consecutive and all-comers patients with a diagnosis of ACS in tertiary care hospitals with on-site catheterisation team 24/7 ('hub').

\section{Patients eligibility}

Inclusion criteria

For inclusion in the study subjects should be diagnosed with ACS according to either of the following two categories, ST elevation acute coronary syndromes (STEACS) or non-ST elevation acute coronary syndromes (NSTEACS), defined as follows:

- Patients with STEACS: symptoms suspected for myocardial ischaemia and persistent (ie, $>20 \mathrm{~min}$, if two consecutive ECG tracings available) ST segment elevation in $\geq 2$ contiguous ECG leads.

Patients with STEACS will be prospectively distinguished into those considered for urgent reperfusion versus those who will not (see section on prospective enrolment and figure 1 and online supplemental e-Figure 1).

Note: positive biomarkers of cardiac necrosis (ie, troponin) are not required to confirm the diagnosis. New or presumably new left-bundle branch block at presentation occurs infrequently, may interfere with ST-elevation analysis and should not be considered

\section{A}

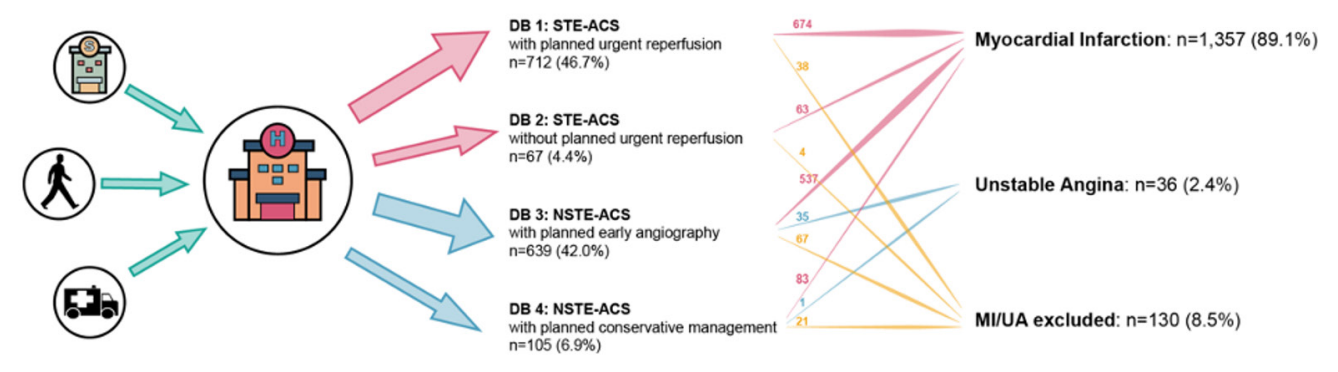

B

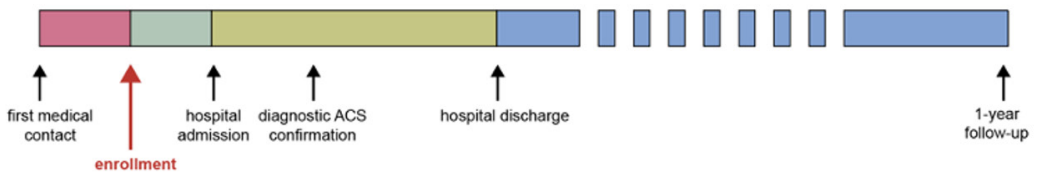

Figure 1 ACS Clinical Governance Programme Structure and Timelines. (A) Eligible patients with ACS could be enrolled from spoke hospitals (transfer), self-presenting to emergency department, or via Emergency Medical System (non-transfer). After inclusion, the ACS population is prospectively divided into four main population. Data are reported from the feasibility, singlecentre phase (1523 patients). (B) Timelines for eligibility and follow-up in relationship to hospitalisation. ACS, acute coronary syndrome; STE-ACS, ST elevation acute coronary syndromes; NSTE-ACS, non-ST elevation acute coronary syndromes; MI, myocardial infarction; UA, unstable angina. 
diagnostic of acute myocardial infarction (AMI) in isolation. ${ }^{11}$

- Patients with NSTEACS: symptoms suspected for myocardial ischaemia without persistent ST elevation in $\geq 2$ contiguous ECG leads.

To be included under NSTEACS category patients should have $\geq 1$ of the following two conditions: (1) ECG evidence of NSTEACS defined as T wave inversion $\geq 1 \mathrm{~mm}(100 \mu \mathrm{V})$ or ST segment depression $\geq 0.5 \mathrm{~mm}$ $(50 \mu \mathrm{V})$ in leads with dominant $\mathrm{R}$ waves and/or (2) biomarker evidence of NSTEACS defined as $\geq$ one positive (ie, above the 99th percentile upper reference limit) troponin value (ie, NSTEMI).

Therefore, troponin-negative patients with NSTEACS (ie, UA pectoris) defined only by isolated symptoms of myocardial ischaemia (ie, negative cardiac biomarkers and normal ECG) are not eligible for inclusion. Patients with NSTEACS will be prospectively distinguished into those considered for a routine invasive management versus those who are not (see section on prospective enrolment and figure 1 and online supplemental e-Figure 1).

\section{Exclusion criteria}

As an all-comers initiative, exclusion criteria will be minimal. Only subjects who, in the opinion of the investigator, are unable to comply with study follow-up procedures, including, but not limited to, patients who are in prison, who are expected to move to a remote country, or who refuse to be followed are to be excluded.

\section{Objectives}

The primary study objective is to systematically measure, in an all-comers population hospitalised for ACS: (1) time to reperfusion in patients with ST elevation ACS and (2) optimal medical therapy at discharge in patients with a final diagnosis of MI or UA.

Secondary objectives broadly include:

- Adherence to a wide range of QIs within multiple domains of care including optimal ACS diagnosis, therapy and individualised risk assessment through monitoring of process of care measures.

- Examine associations of programme participation with trends of QIs adherence over time.

- Monitor the characteristics, treatments and outcomes of patients hospitalised with ACS.

- Explore the association between evidence-based acute treatment strategies and risk-adjusted clinical outcomes.

- Assess utilisation of diagnostic imaging, laboratory tests and invasive procedures, and track hospital/ coronary care unit length-of-stay data.

- Assess trends in medication dosing patterns, and improve drug safety through targeted quality feedback related to medication overdosing.

- Identify barriers to implementing guideline recommendations for patients with AMI, and develop effective strategies to overcome these barriers.

\section{Outcome variables}

Two coprimary efficacy variables as well as secondary and other exploratory variables will be considered.

The two coprimary efficacy variables will be:

1. Proportion of patients with STEACS intended for urgent angiography/reperfusion (ie, DB1) who receive timely reperfusion.

2. Proportion of patients with confirmed MI or UA at discharge who receive optimal medical therapy.

Secondary and exploratory variables include assessment of left ventricular ejection fraction, concordant final diagnosis at discharge, and use of radial access. Outcome definitions and data derivations are listed in the online supplemental appendix.

\section{Clinical outcomes and follow-up procedures}

During the first year of follow-up, the following endpoint will be assessed: death, MI, stroke and bleeding. Patients will be followed for up to 1year for vital status and non-fatal cardiac and cerebrovascular adverse events, including all adjudicated endpoints (see online supplemental appendix).

\section{Programme characteristics}

\section{Prospective enrolment}

The ACS clinical governance programme will enroll patients with an admission diagnosis of ACS prospectively. The prospective nature of the study will regard not only the timing of data collection relative to when the study was designed ${ }^{12}$ but also the diagnostic ACS process. The time of enrolment will be the first objective qualifying ACS criterion, typically the time of diagnostic ECG for patients with STEACS and the time of ECG or troponin (whatever occur first) for patients with NSTEACS. Therefore, unlike other initiatives in patients with ACS, ${ }^{1}$ patients admitted for ACS but discharged with a diagnosis alternative to MI or UA will be included.

The proportion of patients with a discharge diagnosis consistent with ACS (that is MI or UA) as opposed to an alternative diagnosis (such as myocarditis, pericarditis, gastro-oesophageal diseases, etc) will provide insights into the diagnostic processes, indirectly measure its accuracy, and potentially identify early diagnostic gaps.

Four population within the ACS spectrum will be prospectively define based on two stratification questions at the time of inclusion (see online supplemental e-Figure $1)$.

These populations are:

- DB1 (STEACS urgent): patients with STEACS intended for urgent angiography/reperfusion. This population mostly includes STEACS patients for whom primary PCI is intended, typically with symptoms within 12 hours. A minority of this population includes patients with STEACS intended for urgent angiography for persistent ST elevation and/or symptoms but with symptoms onset $>12$ hours, urgent angiography after failed fibrinolysis (rescue PCI), or patients receiving fibrinolysis. 
- DB2 (STEACS not urgent): patients with STEACS NOT intended for urgent angiography/reperfusion. This population mostly includes STEACS patients not receiving reperfusion for late presentation or patient preference. Patients in this category may receive diagnostic angiography for diagnostic and/or prognostic stratification, but not on an urgent basis.

- DB3 (NSTEACS Invasive): patients with NSTEACS intended to be initially managed invasively with coronary angiography, generally within 72 hours. This population includes patients with NSTEACS and high-risk feature (ie, positive troponin, Global Registry of Acute Coronary Events (GRACE) risk score $>140$, haemodynamic/electrical instability) for whom an angiography is initially planned.

- DB4 (NSTEACS Conservative): patients with NSTEACS intended to be managed conservatively. This population mostly includes patients who are considered candidate for an initially conservative strategy. Note that this category may include patients who are subsequently managed with coronary angiography, for recurring symptoms of myocardial ischaemia, or haemodynamic/electrical instability.

\section{Consecutive and inclusive enrolment}

Consecutive patient enrolment is essential to accurately and comprehensively reflect the population of interest. High-risk patients with ACS, such as those with cardiogenic shock and those who die early after presentation, have been generally excluded from clinical studies, typically for a lack of a written informed consent.

In the ACS clinical governance programme, consecutive and broad patient enrolment will be promoted by minimal exclusion criteria and a simplified informed consent process. As a primary quality improvement initiative, ${ }^{13}$ a written informed consent will be required only for a follow-up contact to patients who are discharged alive. Therefore, patients who die in-hospital will be routinely included. Only data collected per standard of care will be considered, which will be anonymised at the level of analysis.

Importantly, consecutive enrolment will be verified and quantified by the concordance with corresponding hospital administrative records using diagnosis-related group (DRG) codes of ACS. Specifically, according to the protocol of the Italian National Agency for Regional Health Services-AGE.NA.S (https://pne.agenas.it/ risultati/protocolli/pro_1.pdf), which is charged to describe hospital-level outcome in Italy, DRG codes related to ACS will be used to identify the totality of hospital-level patients with MI or UA (denominator) against the enrolled patients with a final diagnosis of MI or UA (numerator) as so to quantify the proportion of consecutive patients.

\section{Systematic endpoint adjudication}

The following endpoints-death, MI, stroke, and bleeding — will be systematically adjudicated by a Clinical
Event Committee consisting of at least two physicians according to prespecified criteria. Disagreements will be resolved by consensus or, in case a consensus is not achieved, by a third physician.

All potential events, both reported and unreported by the investigators but identified via case report form (CRF) will be triggered for evaluation. Search strategies for potential endpoints that are not reported by the investigator will be defined via CRF dedicated queries. Other clinical events of interest during (eg, cancer, pulmonary embolism, other medical event requiring unplanned hospitalisation) during the follow-up will be recorded as adverse events but will not undergo formal adjudication. Endpoint definitions are provided in the online supplemental appendix. ${ }^{14}$

\section{Data quality and data sharing policies}

At each site, data entry quality checks will be performed by dedicated staff. Individual patients data will be verified using two primary quality controls: (1) cross review by an external, dedicated MD, (2) statistical cross checks. To facilitate correct data entry, each CRF field has dedicated legend derived by American Heart Association/American College of Cardiology Foundation key data standards. ${ }^{15}$ To promote accessibility, transparency and 'symbiotic' collaborations, ${ }^{16}$ we plan to share individual patient with external investigators on reasonable request according to recommendations the International Committee of Medical Journal Editors. ${ }^{17}$

\section{Principles of study organisation, funding model and current status}

The overarching goal of the programme is to pilot and innovate quality improvement in patient with ACS in Italy to inform a larger, ideally country-based, permanent initiative, such as the Myocardial Ischaemia National Audit Project in the UK. Therefore, a minimalist funding model, that optimise resource utilisation and data quality, is instrumental for the programme purposes. The study has been supported, in part, by a competitive grant: externally sponsored research of AstraZeneca (ESR-16-12480).

After a single-centre (Fondazione IRCCS Policlinico S.Matteo), initial study phase primarily designed to test the feasibility of including a large number of patients with ACS by a dedicated study coordinator ( $\mathrm{n}=1524$ patients enrolled from September 2015 to December 2017), the multicentre phase was implemented in January 2018. As of 10 July 2020, a total of 3388 patients have been enrolled across seven centres, selected across two Italian regions (Lombardia and Emilia Romagna) among those who fulfil the requirements (tertiary care hospitals with on-site catheterisation team 24/7) and were willing to participate. Study termination (last follow-up of last included patient) is expected in September 2021.

During the single-centre phase, we observed (figure 2) an in-hospital death rate of $6 \%$ and a 1-year fatality rate of $10.9 \%$, substantially higher compared with similar ACS registries and randomised controlled trial (RCTs). ${ }^{8} 18$ 

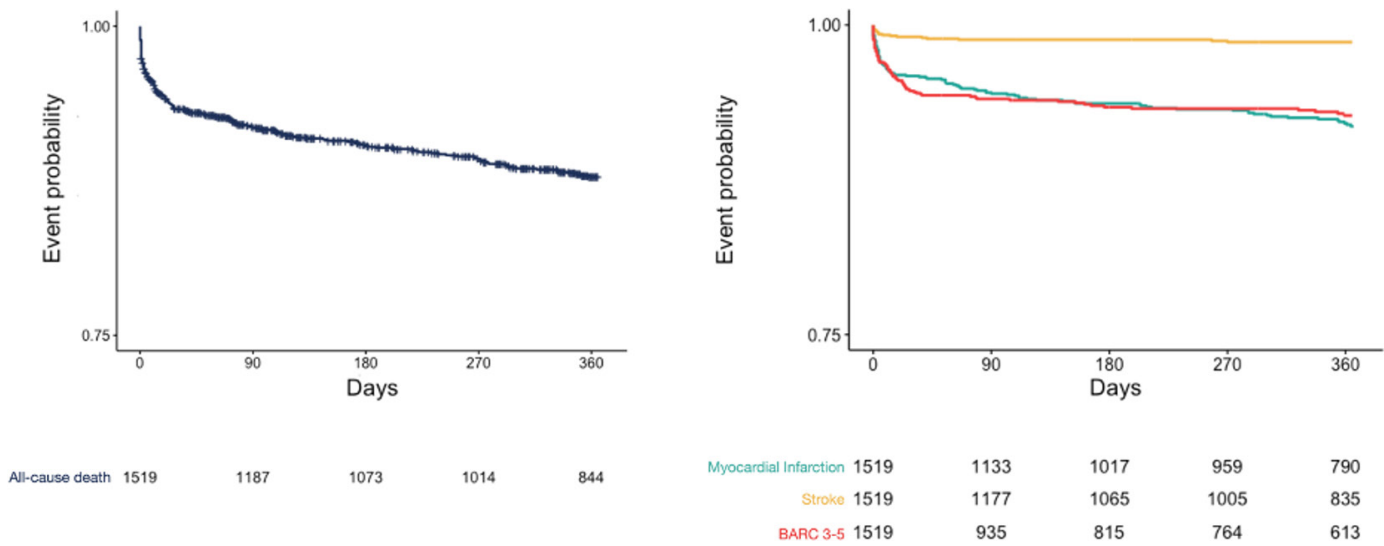

Figure 2 Clinical-event committee adjudicated endpoint rates. The figure report Kaplan-Meier rates for death (left panel) and myocardial infarction (azure), stroke (yellow) and major bleeding (red) on the right panel in the single-centre, feasibility part of the study (1523 patients).

Also, in an analysis including a sample of the calendar year 2016, using the totality of hospital DRG codes, we verified that 573 patients were discharged (dead or alive) from the hospital with a confirmed acute myocardial infarction (AMI, online supplemental e-Figure $2)$. Of these, $474(83 \%)$ were enrolled in the clinical governance programme. The primary reason why the remaining $17 \%$ of patients with confirmed acute MI (ie, 99 patients) were not included was the admission outside a cardiology department (coronary/general intensive care or cardiology ward). Notably, the clinical governance programme allowed to identify 48 patients $(8.4 \%)$ that were not included by administrative AMI codes. ${ }^{19}$

\section{Statistical analysis}

Baseline characteristics will be described using proportions for categorical data, and medians and IQR for continuous data. Adherence to each QIs will be described as proportion with SE or mean value $(95 \% \mathrm{CI})$ as appropriate. To estimate the GRACE risk score adjusted 30 days mortality, we will use predicted probabilities derived from a logistic regression model with 30 days mortality as dependent variable and each patient's calculated GRACE risk score as predictor. To explore the hypothesis that, at the patient-level, QIs adherence is associated with a reduction of clinical endpoint (death and the composite of death, MI or stroke) at 30 days and 1 year, multivariable Cox-regression analysis will be used. Variables included in these models will be prespecified and listed in the online supplemental appendix.

We will use Spearman's ranks to investigate the relationship between all combinations of QIs. Finally, we will also fit a logistic regression model to examine the strength of association between QI measures and 30 days mortality. Using data from the feasibility phase, we estimated an average adherence of $60 \%$ to each of the coprimary QIs (according to preliminary data in OSM Pavia). Therefore, a total of 3090 patients need to be enrolled to have a $90 \%$ power to detect a potential $4 \%$ improvement $(64 \%)$ with a 95\% CI for the multicentre part of the study. Considering possible lost to follow-up and patients with missing data, a final sample of $\approx 3500$ patients is anticipated. In the single centre part, a convenience sample of $\approx 1500$ patients was considered.

\section{ETHICS AND DISSEMINATION}

The clinical governance programme has been approved by local ethics committee of all study sites. As a quality improvement initiative and to promote consecutive inclusion of the population of interest, a written informed consent was required only to patients who were discharged alive. Dissemination will be actively promoted by (1) the registration site (ClinicalTrials.Gov ID NCT04255537), (2) collaboration with other investigators through data sharing.

\section{PATIENT AND PUBLIC INVOLVEMENT}

The clinical Governance Programme in patients with ACS was designed to understand potential gaps in patient care delivery and how to fill these gaps. While patients were not directly involved in the design or conception of the study and no specific patient reported outcome has been considered, the extensive characterisation of several QoC domains is expected to measure and promote patients' health.

\section{DISCUSSION}

Clinical governance is a holistic, patient-centred approach intended to promote a continuous improvement of the quality of the healthcare provided by actively including all individuals involved in a patient's care. ${ }^{20}$ This ambitious vision is highly needed in patients with ACS but require an integration of different care dimensions, including diagnostic and treatments strategies, network organisation and patient satisfaction. Also, to describe an ACS population that accurately reflect the disease of interest, as well as the complex process of care, we designed a study closely integrated with clinical care. This study, inspired by the principles of clinical governance-appropriateness, effectiveness, safety, timeliness, efficiency 
and equity of care as well as patient's centrality-has the ultimate goal to understand, measure and reduce the gaps between scientific evidence and patient care and to inform the design of a subsequent national ACS surveillance system that may contribute to the scientific enquiry process of ACS care and facilitate quality improvement efforts. ${ }^{2122}$

\section{Potential implications of a clinical governance programme in patients with ACS}

Ideally, a quality improvement initiative should benefit (and thus include) the entire population of interest. The inclusion of consecutive patients is also vital to accurately reflect the disease of interest and may have profound, practice-changing implications on the diagnosis, management and treatment strategies of patients with ACS. In the feasibility phase, the in-hospital and 1-year mortality rate we observed $-6 \%$ and $10.9 \%$, respectively-it is from twofold to threefold higher than current all-comers ACS registries and RCTs. ${ }^{818}$ Even considering large global ACS population of 10-20 years ago, the in-hospital mortality we observe remains substantially higher. ${ }^{23}{ }^{24}$ This difference underscores potential gap in outcome verification of current registries and how initiatives that abridge in part a written consent, especially for observational studies, may help the identification of a consecutive, truly representative, population with ACS.

Importantly, metrics that verify and quantify the degree of consecutive inclusion may be helpful in this context. In a 1-year analysis during the initial feasibility phase, ${ }^{19}$ we verified that $83 \%$ of the totality of patients with AMI admitted to one of the participating Institution were enrolled, indicating a benchmark to the ideal, complete consecutive sample. Patients who were not included (17\%) were typically managed conservatively outside cardiology, indicating the need to better identify and include these high-risk (often forgotten) patients as so to better inform quality improvement efforts. Importantly, administrative criteria showed modest diagnostic accuracy for AMI diagnosis (mostly due to obsolete and nondiscriminating ICD-9 diagnostic codes), and 8.4\% (48) patients discharged with a confirmed acute MI were only identified via this initiative, due to DRG misclassification of acute MI codes. This further highlight how such initiatives might contribute to hospital-level improvement in diagnostic verification and accuracy.

We acknowledge the prospective nature of ACS, an admission diagnosis that was originally intended to triage patients with persistent ST elevation at the ECG to immediate reperfusion. Notably, $8.5 \%$ of patients initially admitted with an operational diagnosis of ACS were eventually discharged with a diagnosis alternative to AMI or UA pectoris, highlighting opportunities to refine the diagnostic process in approximately 1 in 12 patients. It is well known that a relevant proportion-from $5 \%$ to $10 \%$-of patients with ACS will ultimately have an alternative cardiac or non-cardiac diagnosis. ${ }^{25-27}$ Therefore, identification of patients with alternative diagnoses is necessary to improve diagnostic accuracy of ACS criteria and minimise potentially inappropriate treatments.

Finally, this initiative could provide a useful structure for pragmatic RCTs, such as those of the Swedish Websystem for Enhancement and Development of Evidencebased care in Heart disease Evaluated According to Recommended Therapies (SWEDEHEART) initiative, ${ }^{28}$ with minimal additional costs.

\section{Study limitation}

We acknowledge some limitations. One limitation is the selective inclusion of patients from a limited number of tertiary care hospitals. Also, similar to other indicatives, detailed indicators of patient-related delays (such as the activation of emergency medicine system, onset time of most recent symptoms) are challenging to standardise and will not be collected.

\section{CONCLUSIONS}

The clinical governance programme in patients with ACS is a pilot, quality improvement initiative closely integrated with clinical care that may promote quality measurement and improvement, better understanding of the disease process of care, scientific discovery, as so to ultimately improve the prognosis of patients with ACS.

\section{Author affiliations}

${ }^{1}$ Cardiology, University of Pavia, Pavia, Lombardia, Italy

${ }^{2}$ Fondazione IRCCS Policlinico San Matteo, Pavia, Lombardia, Italy

${ }^{3}$ Maggiore Hospital Carlo Alberto Pizzardi, Bologna, Emilia-Romagna, Italy

${ }^{4}$ Ospedale degli Infermi di Rimini, Rimini, Emilia-Romagna, Italy

${ }^{5}$ Ospedale Morgagni-Pierantoni, Forli, Emilia-Romagna, Italy

${ }^{6}$ Maria Cecilia Hospital SpA, Cotignola, Emilia-Romagna, Italy

${ }^{7}$ UO Cardiologia, Azienda Ospedaliero Universitaria di Ferrara Arcispedale

Sant'Anna, Cona, Italy

${ }^{8}$ IRCCS MultiMedica, Sesto San Giovanni, Lombardia, Italy

${ }^{9}$ Ospedale Santa Maria delle Croci, Ravenna, Emilia-Romagna, Italy

${ }^{10}$ Azienda Ospedaliero Universitaria Città della Salute e della Scienza di Torino, Torino, Piemonte, Italy

Twitter Gianluca Campo @GianlucaCampo78

Acknowledgements The authors thank Daniela Civardi for the editorial support in preparing this manuscript. They also thank all the medical students, residents and fellows supporting the study coordinator in data entry and validation: Alberto Barengo, Chiara Castelli, Manuel Walker, Sebastiano Carli, Francesco Alfio Russo, Francesco Matteo Dioniso.

Contributors SL designed the study, acquired the funding, and wrote the first draft of the manuscript. All the remaning authors acquired the data and critically revised the manuscript for substantial. All authors gave final approval for this version of the manuscript to be submitted for publication.

Funding The study has been supported, in part, by a competitive grant: externally sponsored research of AstraZeneca (ESR-16-12480).

Competing interests SL reports personal fees for advisory board participation from AstraZeneca, Chiesi, BMS/Pfizer, Novo Nordisk, and The Medicine Company, outside the submitted work.

Patient consent for publication Not required.

Provenance and peer review Not commissioned; externally peer reviewed.

Data availability statement Data are available upon reasonable request. To promote accessibility, transparency, and "symbiotic" collaborations (reference 16) we plan to share individual patient with external investigators upon reasonable request according to recommendations from the International Committee of Medical Journal Editors. 
Open access This is an open access article distributed in accordance with the Creative Commons Attribution Non Commercial (CC BY-NC 4.0) license, which permits others to distribute, remix, adapt, build upon this work non-commercially, and license their derivative works on different terms, provided the original work is properly cited, appropriate credit is given, any changes made indicated, and the use is non-commercial. See: http://creativecommons.org/licenses/by-nc/4.0/.

ORCID iDs

Sergio Leonardi http://orcid.org/0000-0002-4800-6132

Gianluca Campo http://orcid.org/0000-0002-5150-188X

\section{REFERENCES}

1 Peterson ED, Roe MT, Chen AY, et al. The NCDR action RegistryGWTG: transforming contemporary acute myocardial infarction clinical care. Heart 2010;96:1798-802.

2 Jernberg T, Attebring MF, Hambraeus K, et al. The Swedish Websystem for enhancement and development of evidence-based care in heart disease evaluated according to recommended therapies (SWEDEHEART). Heart 2010;96:1617-21.

3 Herrett E, Smeeth L, Walker L, et al. The myocardial ischaemia national audit project (MINAP). Heart 2010;96:1264-7.

4 Shahian DM, Normand S-LT. Comparison of "risk-adjusted" hospital outcomes. Circulation 2008;117:1955-63.

5 Schiele F, Gale CP, Bonnefoy E, et al. Quality indicators for acute myocardial infarction: a position paper of the acute cardiovascular care association. Eur Heart J Acute Cardiovasc Care 2017;6:34-59.

6 Bebb O, Hall M, Fox KAA, et al. Performance of hospitals according to the ESC ACCA quality indicators and 30-day mortality for acute myocardial infarction: national cohort study using the United Kingdom myocardial ischaemia national audit project (MINAP) register. Eur Heart J 2017;38:974-82.

7 Rossello X, Medina J, Pocock S, et al. Assessment of quality indicators for acute myocardial infarction management in 28 countries and use of composite quality indicators for benchmarking. Eur Heart J Acute Cardiovasc Care 2020:204887262091185.

8 De Luca L, Leonardi S, Cavallini C, et al. Contemporary antithrombotic strategies in patients with acute coronary syndrome admitted to cardiac care units in Italy: the EYESHOT study. Eur Heart $J$ Acute Cardiovasc Care 2015;4:441-52.

9 Olivari Z, Steffenino G, Savonitto S, et al. The management of acute myocardial infarction in the cardiological intensive care units in Italy: the 'BLITZ 4 Qualità' campaign for performance measurement and quality improvement. Eur Heart J 2012;1:143-52.

10 Di Chiara A, Chiarella F, Savonitto S, et al. Epidemiology of acute myocardial infarction in the Italian CCU network: the blitz study. Eur Heart J 2003:24:1616-29.

11 O'Gara PT, Kushner FG, Ascheim DD, et al. 2013 ACCF/AHA guideline for the management of ST-elevation myocardial infarction: a report of the American College of cardiology Foundation/American heart association Task force on practice guidelines. J Am Coll Cardiol 2013;61:e78-140.

12 Vandenbroucke JP, von Elm E, Altman DG. Strengthening the reporting of observational studies in epidemiology (STROBE): explanation and elaboration. Ann Intern Med 2007;147:W-94.

13 Miller FG, Emanuel EJ. Quality-Improvement research and informed consent. N Engl J Med 2008;358:765-7.
14 Hicks KA, Mahaffey KW, Mehran R, et al. 2017 cardiovascular and stroke endpoint definitions for clinical trials. J Am Coll Cardiol 2018;71:1021-34.

15 Cannon CP, Brindis RG, Chaitman BR, et al. 2013 ACCF/AHA key data elements and definitions for measuring the clinical management and outcomes of patients with acute coronary syndromes and coronary artery disease: a report of the American College of cardiology Foundation/American heart association Task force on clinical data standards (writing Committee to develop acute coronary syndromes and coronary artery disease clinical data standards). $J$ Am Coll Cardiol 2013;61:992-1025.

16 Longo DL, Drazen JM. Data sharing. N Engl J Med 2016;374:276-7.

17 Loder E, Groves T. The BMJ requires data sharing on Request for all trials. BMJ 2015;350:h2373.

18 Valgimigli M, Frigoli E, Leonardi S, et al. Radial versus femoral access and bivalirudin versus unfractionated heparin in invasively managed patients with acute coronary syndrome (matrix): final 1 -year results of a multicentre, randomised controlled trial. Lancet 2018;392:835-48.

19 Barengo A, Portolan M, Raisaro A, et al. Lack of diagnostic accuracy of acute myocardial infarction discharge codes by Italian national agency:insight from a quality improvement initiative in a tertiary care hospital. Eur Heart J Acute Cardiovasc Care 2019;8:5-440.

20 Veenstra GL, Ahaus K, Welker GA, et al. Rethinking clinical governance: healthcare professionals' views: a Delphi study. BMJ Open 2017; 7:e012591.

21 Jernberg T, Attebring MF, Hambraeus K, et al. The Swedish websystem for enhancement and development of evidence-based care in heart disease evaluated according to recommended therapies (SWEDEHEART). Heart 2010;96:1617-21.

22 Wallentin L, Gale CP, Maggioni A, et al. EuroHeart: European unified registries on heart care evaluation and randomized trials. Eur Heart $J$ 2019:40:2745-9.

23 McNamara RL, Kennedy KF, Cohen DJ, et al. Predicting in-hospital mortality in patients with acute myocardial infarction. J Am Coll Cardiol 2016;68:626-35.

24 Steg PG, Goldberg RJ, Gore JM, et al. Baseline characteristics, management practices, and in-hospital outcomes of patients hospitalized with acute coronary syndromes in the global registry of acute coronary events (grace). Am J Cardiol 2002;90:358-63.

25 De Ferrari GM, Fox KAA, White JA, et al. Outcomes among nonST-segment elevation acute coronary syndromes patients with no angiographically obstructive coronary artery disease: observations from 37,101 patients. Eur Heart J Acute Cardiovasc Care 2014;3:37-45.

26 De Ferrari GM, Leonardi S, Baduena L, et al. Patients with acute coronary syndrome and nonobstructive coronary artery disease in the real world are markedly undertreated. $J$ Cardiovasc Med 2011;12:700-8.

27 Planer D, Mehran R, Ohman EM, et al. Prognosis of patients with non-ST-segment-elevation myocardial infarction and nonobstructive coronary artery disease: propensity-matched analysis from the acute catheterization and urgent intervention triage strategy trial. Circ Cardiovasc Interv 2014;7:285-93.

28 Wachtell K, Lagerqvist B, Olivecrona GK, et al. Novel trial designs: lessons learned from thrombus aspiration during ST-segment elevation myocardial infarction in Scandinavia (taste) trial. Curr Cardiol Rep 2016;18:11. 